\title{
Negative effects of diabetes-related distress on health-related quality of life: an evaluation among the adult patients with type 2 diabetes mellitus in three primary healthcare clinics in Malaysia
}

\author{
Boon-How Chew ${ }^{1 *}$, Sherina Mohd-Sidik ${ }^{2}$ and Sazlina Shariff-Ghazali ${ }^{1}$
}

\begin{abstract}
Background: Patients with type 2 diabetes mellitus (T2D) often experienced change in life, altered self-esteem and increased feelings of uncertainty about the future that challenge their present existence and their perception of quality of life (QoL). There was a dearth of data on the association between diabetes-related distress (DRD) and health-related quality of life (HRQOL). This study examined the determinants of HRQoL, in particular the association between DRD and HRQoL by taking into account the socio-demographic-clinical variables, including depressive symptoms (DS) in adult patients with T2D.

Methods: This cross-sectional study was conducted in 2012-2013 in three public health clinics in Malaysia. The World Health Organization Quality of Life-Brief (WHOQOL-BREF), 17-items Diabetes Distress Scale (DDS-17), and 9-items Patient Health Questionnaire (PHQ-9) were used to measure HRQoL, DRD and DS, respectively. The aim of this research was to examine the association between the socio-demographic-clinical variables and HRQoL as well as each of the WHOQOL-BREF domain score using multivariable regression analyses.

Results: The response rate was $93.1 \%$ (700/752). The mean (SD) for age was 56.9 (10.18). The majority of the patients were female $(52.8 \%)$, Malay $(53.1 \%)$ and married (79.1\%). About $60 \%$ of the patients had good overall HRQoL. The mean (SD) for Overall QoL, Physical QoL, Psychological QoL, Social Relationship QoL and Environmental QoL were 61.7 (9.86), 56.7 (10.64), 57.9 (11.73), 66.8 (15.01) and 65.3 (13.02), respectively. The mean (SD) for the total DDS-17 score was 37.1 (15.98), with $19.6 \%$ (136/694) had moderate distress. DDS-17 had a negative association with HRQOL but religiosity had a positive influence on HRQoL (B ranged between 3.07 and 4.76). Women, especially younger Malays, who had diabetes for a shorter period of time experienced better HRQoL. However, patients who were not married, had dyslipidaemia, higher levels of total cholesterol and higher PHQ-9 scores had lower HRQoL. Macrovascular complications showed the largest negative effect on the overall HRQoL (adjusted B $=-4.98$, $95 \% \mathrm{Cl}-8.56$ to -1.40$)$.

\footnotetext{
* Correspondence: chewboonhow@gmail.com

'Department of Family Medicine, Faculty of Medicine and Health Sciences, Universiti Putra Malaysia, 43400 Serdang, Selangor, Malaysia

Full list of author information is available at the end of the article
} 
(Continued from previous page)

Conclusion: The majority of primary care adult with T2D had good overall HRQoL. Furthermore, the independent determinants for HRQoL had also concurred with many past studies. In addition, the researchers found that DRD had negative effects on HRQoL, but religiosity had positive influence on HRQoL. Appropriate support such as primary care is needed for adult patients with T2D to improve their life and their HRQoL.

Trial registration: NMRR-12-1167-14158

Keywords: Quality of life, Distress, Depression, Type 2 diabetes mellitus, Primary care, Religious beliefs

\section{Background}

Type 2 diabetes mellitus (T2D) has been known to have changed life experiences, altered self-esteem, challenged present existence and increased uncertainty about the future [1-8].

The lives of individuals would change from the moment when they experience the symptoms such as chronic hyperglycaemia, weight loss, skin infections and lethargy right up to the diagnosis of diabetes mellitus. A complete change in life routines continues from the demands of more regular healthy life-styles, adherence to daily medication and scheduled visits to various types of healthcare professionals. Repeated physical examinations, laboratory tests and psychosocial counselling reverberating the ideas of risky threats to bodily health and the long-term complications [9] could reduce the self-esteem and selfconfidence that inspire adventures and motivation in the patients' lives [10, 11]. Family members and friends would also avoid discussing the implications of the disease with the patients $[12,13]$. There might be occasional feelings of stigmatisation at family gatherings or even at work-related social functions [7, 14]. Left alone to these physical and psychological onslaughts, many patients may feel overwhelmed, shaken and questioned their personal values and beliefs of the purpose of self-regulation [10], self-care and self-management [15]. Hope for the future may be replaced with dread of complications from the disease and the adverse effects of medications which may result in frequent and disruptive effects such as restlessness, distress, anxiety, and depression [3, 16]. Eventually, all of these negative emotions may lead to the failure of adherence to health recommendations, medications, medical follow-ups $[17,18]$, and may increase the number of medical leaves [16]. Consequently, patients' personal perceptions turn from bad to worse, and they start to smoke excessively [19], practise uncontrolled diet, and have conflicts with their significant others [20]. This would cause the symptoms of diabetes and their associated complications to return despite escalating their medications [21].

Quality of life (QoL) of the patients with diabetes mellitus represents personal perceptions of life experience, social, vocational and domestic functioning against hope and ideals from aspects of physical, psychological, relationships, environmental and spiritual domains [22, 23].
A large body of literature has focused on the impacts of diabetes mellitus of both type 1 and type 2 on patients, albeit in vast aspects and contexts of life [4, 21, 24], and the associations with QoL, self-efficacy and disease control for adult patients with T2D. Moreover, patients who perceived higher levels of QoL also showed that they had better social support, acceptance of the seriousness and consequences of the disease, and had less difficulty in managing their diabetes [25]. In spite of the many different QoL scales used in past studies, there are similar effects of socio-demographic variables and clinical parameters on the domains of QoL across the globe [18, 26-34]. However, there was a dearth of data on the association between diabetes-related distress (DRD) and health-related quality of life (HRQoL). Accordingly, the purpose of this study was to evaluate the determinants of HRQoL, particularly to examine the effects of DRD on HRQoL taking into account other socio-demographic-clinical variables of adult patients with T2D who received regular primary medical care in three public health clinics in Malaysia. Hence, the research question was: what are the independent effects of socio-demographic-clinical variables, including DRD and depressive symptoms (DS), on HRQoL in adult patients with T2D?

\section{Methods}

\section{Ethics, consent and permission}

This cross-sectional study was approved by the Medical Research Ethics Committee (MREC), Malaysia's Ministry of Health with the reference number NMRR-12-116714158. Respondents had provided their written informed consent before participating in the study, and the anonymity of the patients was preserved throughout the study.

\section{Setting}

Participants were recruited from three public health clinics: Seri Kembangan Health Clinic (SK), Dengkil Health Clinic (DK) and Salak Health Clinic (SL) in Malaysia. These health clinics were chosen because they were different in terms of the patients' characteristics and geographical regions. SK is an urban clinic located in the vicinity of the Chinese community, and thus it is visited mainly by Chinese patients. DK is a rural clinic frequented mostly by 
Indians in a larger proportion than a usual public health clinic. SL is a rural clinic situated in a Malay-majority residential area. The variability of the sites provided a broad range of patients on which the associations of DRD, HRQoL and socio-demographic clinical parameters can be assessed. The purpose of this report was to determine the associations of the independent variables and the dependent variables irrespective of the health clinics.

\section{Patient}

\section{Definitions of study participants}

The researchers had sampled consecutively all the patients with T2D who came to the clinics. The patients were at least 30 year-old and were diagnosed with T2D for more than a year. The patients who were recruited for this study fulfilled the following criteria: have been on regular follow-ups, had at least three visits at the clinic in the past 1 year and had recent blood test results done within the past 3 months. Patients who were pregnant or lactating, had psychiatric/psychological disorders that could impair judgment and memory, and could not read or understand English, Malay or Mandarin were excluded from this study. Patients who fulfilled the criteria were approached and informed of the study and their written consents were obtained before the questionnaires were administered in their preferred language. Trained research assistants also interviewed the patients who were not able to answer the questionnaires themselves.

Besides a questionnaire on demography (age, gender, ethnicity, religion, educational level, occupation, monthly income, self-perceive social support) and smoking status, a structured case record form was used to document the patients' history which includes co-morbidities (hypertension and hyperlipidaemia/dyslipidaemia), diabetes-related complications, duration of the diabetes, $\mathrm{HbAlc}$, blood pressure, lipid profiles, number and type of medication used. Three questionnaires were distributed to evaluate HRQoL, DRD and DS, and they were prepared in three languages: English, Malay and Mandarin.

\section{Definitions of diseases}

The definition of T2D was when their case records fulfilled any of these criteria: (i) either having documented a diagnosis of diabetes mellitus according to the 1999 World Health Organization criteria [35] or (ii) currently treated with lifestyle modifications, oral anti-hyperglycaemic agents or insulin. Hypertension was diagnosed if the systolic blood pressure was $\geq 130 \mathrm{~mm} \mathrm{Hg}$ and/or the diastolic blood pressure was $\geq 80 \mathrm{~mm} \mathrm{Hg}$ on two of the successive readings obtained by the clinic's physicians. A blood pressure $(\mathrm{BP})<130 / 80 \mathrm{mmHg}$ was regarded as controlled BP, and this was the mean of the two readings in the rested position with the arm positioned at the heart level, using a cuff of an appropriate size. Hyperlipidaemia refers to an increase in the concentration of one or more plasma or serum lipids, usually caused by cholesterol and triglycerides, and the term dyslipidaemia was used for either an increase or decrease in the concentration of one or more plasma or serum lipids [a low density lipoproteincholesterol $(\mathrm{LDL}-\mathrm{C})>2.6 \mathrm{mmol} / \mathrm{L}$, triglyceride $(\mathrm{TG})>$ $1.7 \mathrm{mmol} / \mathrm{L}$ and high density lipoprotein-cholesterol $(\mathrm{HDL}-\mathrm{C})<1.1 \mathrm{mmol} / \mathrm{L}]$. Body mass index (BMI) was calculated as weight was divided by height squared. A LDL$\mathrm{C} \leq 2.6 \mathrm{mmol} / \mathrm{L}$ and $\mathrm{HbA} 1 \mathrm{c} \leq 6.5 \%$ were regarded as the other treatment targets [36, 37]. These clinical data were retrieved from the patient's medical records using a case record form on the same day when the patient completed the questionnaires.

\section{Definitions of diabetes-related complications}

There were five diabetes-related complications in this study. Three were classified as microvascular complications (MicroCx) which comprised retinopathy, nephropathy, and diabetic foot problems (DFP). Another two complications were classified as macrovascular complications (MacroCx) which comprised ischemic heart disease and cerebrovascular disease, or stroke. These complications were retrieved from the patient's medical records. Diagnoses of these complications were made or confirmed by the attending physicians at the clinics based on the medical symptoms, laboratory results, radiological evidence, and treatment history obtained from the clinic and other hospitals. Nephropathy was diagnosed if any of the following was present: microalbuminuria, proteinuria, serum creatinine $>150 \mathrm{mmol} / \mathrm{L}$ or estimated glomerular filtration rate $<60 \mathrm{mls} / \mathrm{min}$ (calculated using CockroftGault formula) persisted ( $\geq 2$ occasions with at least 3 months apart). DFP comprised foot deformity, current ulcer, amputation, peripheral neuropathy, or peripheral vascular disease.

\section{Instruments}

\section{Health-related quality of life}

The World Health Organization Quality of Life- Brief (WHOQOL-BREF) was chosen in this study as the HRQoL measure. Although it is not diabetes-specific, it is relevant to people with diabetes [22]. The WHOQOLBREF was developed internationally, cross-culturally and produces four HRQoL domains [38]. The four domain scores denote an individual's perception of HRQoL in the following domains: Physical (PQOL), Psychological (YQOL), Social Relationships (SRQOL) and Environment (EQOL). The sum of these domain scores produces the overall quality of life (OQOL) [39]. This fourdomain structure has the comparative fit index of 0.901, which demonstrates a good internal consistency with Cronbach alpha values for each of the four domain scores which ranged from 0.66 (for domain 3 ) 
to 0.84 (for domain 1) [39, 40]. There are also two items on overall perceptions of HRQoL that are examined separately. Question 1 asks about the individual's overall perception of HRQoL, and question 2 asks about the individual's overall perception of his or her health.

Each item, from item number 3 to 26, contributes equally to their domain score. After the negatively framed constituent questions were reverse scored, domain scores were calculated by computing the mean of the item scores within the domain. The mean domain scores were then multiplied by 4 providing a range of 4 to 20 and transformed to 100 ([score - 4] " 100/16) in further analyses. The sum of the domain scores were scaled in a positive direction (i.e. higher scores denote higher HRQoL). Where up to two items were missing, the mean of the other items in the domain were substituted. Where more than $20 \%$ of the data were missing from an assessment, the assessment was discarded. Where more than two items were missing from the domain, the domain score was not calculated (with the exception of domain 3, where the domain was only calculated if $<1$ item was missing). There were high correlations between the domain scores based on the WHOQOL-100 and domain scores calculated using items in the WHOQOL-BREF [39]. These correlations ranged from 0.89 (for domain 3) to 0.95 (for domain 1). The WHOQOL-BREF was shown to be comparable to the WHOQOL-100 which could discriminate between the ill and well groups [38, 39].

\section{Diabetes-Related Distress (DRD)}

The 17-item Diabetes Distress Scale (DDS-17) assesses problems and hassles concerning diabetes in the past 1 month on a Likert scale which had measurements of 1 (not a problem) to 6 (a very serious problem) [41]. The total DDS-17 score ranges from 17 to 102 . The score was calculated by summing up the patient's responses to the appropriate items and divided by the number of items in that scale. A mean item score of $\geq 3$ (moderate distress) is considered a level of distress worthy of clinical attention. The DDS-17 has been found to have adequate and better psychometric properties compared to other similar scales [42]. A local translation and validation study of the Malay version of DDS-17 showed a high internal consistency (Cronbach's $\alpha=0.94$ ) and testretest reliability value of $0.33(P=0.009)$ [43]. There was a significant relationship between the mean DDS-17 item score categories $(<3$ vs $\geq 3)$ and HbAlc categories $(<7 \%$ vs $\geq 7 \%)\left(X^{2}=4.20 ; P=0.048\right)$. The DDS-17 sensitivity and specificity, with positive and negative predictive values were $56.5,23.8,7.6$ and $83.1 \%$, respectively. A Chinese version of the DDS-17 was found to have good psychometric properties with Cronbach's $\alpha$ of 0.90 for internal consistency and test-retest reliability coefficient of 0.74 [44].

\section{Depressive Symptoms (DS)}

The Patient Health Questionnaire (PHQ-9) has been known to have a good construct and criterion validity in making diagnosis and assessing the severity of DS, of which a total score of $\geq 10$ indicates a sensitivity of $88 \%$ and a specificity of $89 \%$ for major DS [45]. The PHQ-9 refers to the symptoms experienced by patients during the 2 weeks prior to answering the questionnaire (for example thoughts that you would be better off dead or of hurting yourself in some way). The PHQ-9 scores range from 0 to 27, and each of the nine items has a score starting from 0 (not at all) to 3 (nearly every day). PHQ-9 scores of $0-4,5-9,10-14,15-19$, and 20-27 represent none to minimal, mild, moderate, moderately severe, and severe DS, respectively. The Malay version of PHQ-9 had been locally validated with a sensitivity of $87 \%$ (95\% confidence interval 71 to $95 \%$ ), a specificity of $82 \%$ (74 to $88 \%$ ), positive likelihood ratio (LR) 4.8 (3.2, 7.2) and negative LR $0.16(0.06,0.40)$ [46]. DS was included in this study as one of the main covariates of DRD [47]. The Chinese version of the PHQ-9 is reported to have good psychometric properties with an internal consistency of 0.82 and test-retest reliability of 0.76 over a 2-week interval [48].

\section{Statistical analyses}

A sample size was calculated using GPower 3.1.2 software with an estimated effect size at 1.5 [47, 49], of DRD on HRQoL, with a power of 0.95 and significance at 0.05 ; the estimated sample size was 500 . Taking into consideration the fact that $30 \%$ of the data in the patient's medical records were either incomplete or missing, and $30 \%$ of the data in the questionnaires filled by the patients were incomplete, and the sample size needed was 650 .

Quantitative data analyses were then executed with IBM SPSS Statistics version 21.0. Comparisons of mean levels were performed using the Student's t-test for unpaired samples and Chi square test was used for proportionate samples. If the value of $P<0.05$, it was considered to be significant at two tails. Independent variables include the demographic, DRD (measured by DDS-17), DS (measured by PHQ-9), and clinical data.

The researchers had observed that the sociodemographic characteristic was according to the tertile of the overall quality of life (OQOL). Visual inspections on the histogram and the statistical tests confirmed the normal distributions of OQOL and the four domain scores (PQOL, YQOL, SRQOL and EQOL). Then the relationship between the independent variables towards OQOL and each of the domain score were examined using multiple linear regression analyses.

Univariable analyses were executed for all the studied variables and the variables that showed significant effects 
on the WHOQOL-BREF scores were included in the final multiple linear regression analyses. Socio-demographic data that were continuous and ordinal were entered in the linear regression while the nominal variables were transformed to binary (such as ethnicity, religion and marital status). Employment status variable was re-ordered into three categories: unemployed (0), retired/home manager (1) and employed (2) based on the initial assessments of the direction of the effect on WHOQOL-BREF scores. Clinical variables that had intervals were first entered in the linear regression. The absence of association prompted the entry of the clinical variables to be in a binary nature in accordance to the recommended targets of control (1). MicroCx and MacroCx were preferentially entered in sequence in the linear regression analyses instead of the combined variable of any diabetes complications which was only entered when both the MicroCx and MacroCx were found not in association with HRQoL. This was done so that the individual effects of MicroCx or MacroCx could be discovered, and the combined variable for both of these complications would confirm the true state of no association with a larger sample size.

The multicollinearity between the variables was checked with a correlation matrix and an inspection of their standard error (SE) magnitude. In this study, no variables correlated with each other, $r<0.2$ and SEs were all within 0.001 to 5.0. In all the final models, Q-Q plots gave an indication of normality, the residual plots indicated a fulfilment of linearity and homogeneity assumptions. Age, gender and ethnicity were included in all the models because these three variables were potential confounders.

\section{Results}

The participants' response rate was $93.1 \%$ (700/752). Out of these respondents, 694, 700, 697 and 698 had complete data on summed OQOL, PQOL, YQOL, SRQOL and EQOL, respectively. The mean (SD) for age was 56.9 (10.18) years. More than half were women (52.8 \%) and Malay (53.1 \%) (Table 1). Furthermore, the majority of the respondents were either married or living with partners (79.1 \%), had non-tertiary education (89.0\%), and were earning < RM 3000 (about USD 850) per month (94.4\%). Most patients did some exercise $(57.6 \%)$ and never smoked (76.1\%). About $80 \%$ of the patients reported that they had hypertension compared to about $40 \%$ who had dyslipidaemia. Patients who used oral hypoglycaemic (OHA), anti-hypertensive agents (AHA), and lipidlowering agents (LLA) were about $91 \%, 88 \%$ and $77 \%$, respectively (Table 1).

There were only a minority of patients who achieved treatment targets. About $10 \%$ attained $\mathrm{BMI}<23 \mathrm{kgm}^{-2}$,
$15 \%$ achieved HbA1c $<6.5 \%, 25 \%$ achieved HbA1c $<$ $7.0 \%, 30 \%$ attained BP $\leq 130 / 80 \mathrm{mmHg}$ and $40 \%$ attained LDL-C $\leq 2.6 \mathrm{mml} / \mathrm{L}$ (Table 1 ). In addition, about $12 \%$ had one of the diabetes-related complications, and this rate seemed to parallel with the number of patients who were on anti-platelet agents (Table 1).

Based on the first two items of the WHOQOL-BREF, a majority of the adult patients with T2D at the primary care clinics had good HRQoL. About $60 \%$ of the respondents had more than good overall HRQoL and were satisfied with their health (Fig. 1). The mean (SD) for OQOL, PQOL, YQOL, SRQOL and EQOL were 61.7 (9.86), 56.7 (10.64), 57.9 (11.73), 66.8 (15.01) and 65.3 (13.02), respectively. The patterns of distribution for WHOQOL-BREF domains in the three participating clinics were largely similar with PQOL and YQOL at the trough, and SRQOL and EQOL at the peak (Fig. 2). Patients from SL health clinic showed a significantly higher HRQoL. This association disappeared after the other socio-demographic variables were adjusted in the multivariable analyses (Table 2).

\section{Socio-demographic profile and quality of life}

Male patients with T2D were generally experienced lower HRQoL except in PQOL and significantly so in SRQOL (Table 2). However, Malay patients had significantly better EQOL except in PQOL and YQOL (Table 2). Patients who were older and had diabetes for a longer duration had negative impacts on HRQoL in almost all of the sub-domains which was statistically significant with the SRQOL domain (Table 2). We observed that being religious was a socio-demographic variable that had the largest positive and independent effects on OQOL $(B=3.07)$, PQOL $(B=3.22)$ and YQOL $(B=4.76)$ (Table 2). Also, being employed as compared to those unemployed and retired, was a significant independent determinant of OQOL $(\mathrm{B}=2.08)$ and PQOL $(\mathrm{B}=2.34)$. Patients who exercised more frequently per week perceived higher OQOL (adjusted $B=1.31$ ), SRQOL (adjusted $B=2.38$ ), and EQOL (adjusted $\mathrm{B}=1.99$ ).

\section{Clinical parameters and quality of life}

Having co-morbidities and diabetes-related complications would reduce WHOQOL-BREF scores (Table 2). Dyslipidaemia was shown to have significant independent negative impacts on YQOL $(B=-3.14)$ and EQOL $(B=-2.78)$. Although hypertension showed a positive effect on SRQOL (Table 2), MacroCx caused consistent negative and independent effects on HRQoL, OQOL $(\mathrm{B}=-4.98,95 \% \mathrm{CI}-8.56$ to -1.40$)$ and EQOL $(\mathrm{B}=-4.38$, $95 \% \mathrm{CI}-8.30$ to -0.46$)$.

In general, clinical biomarkers of disease condition and control had negative associations with HRQoL perception. The examples include HDL-C on PQOL 
Table 1 The socio-demographic and clinical characteristics according to the tertile of the World Health Organization Quality of Life- Brief (WHOQOL-BREF)

\begin{tabular}{|c|c|c|c|c|c|c|c|}
\hline & & \multicolumn{4}{|c|}{ Tertile WHOQoL-BREF total score, $n(\%)$} & \multirow[t]{2}{*}{$x^{2}$} & \multirow[t]{2}{*}{$P^{*}$} \\
\hline & & Total ${ }^{a}$ & Low $^{b}$ & Intermediate $^{\mathrm{b}}$ & High $^{\mathrm{b}}$ & & \\
\hline \multirow[t]{3}{*}{ Health Clinic } & Seri Kembangan & $218(31.4)$ & $79(36.2)$ & $75(34.4)$ & $64(29.4)$ & 12.40 & 0.015 \\
\hline & Dengkil & $123(17.7)$ & $43(35.0)$ & $50(40.7)$ & $30(24.4)$ & & \\
\hline & Salak & $353(50.9)$ & $107(30.3)$ & $107(30.3)$ & $139(39.4)$ & & \\
\hline \multirow[t]{3}{*}{ Age, year } & $\leq 50$ & $186(26.9)$ & $62(33.3)$ & $52(28.0)$ & $72(38.7)$ & 5.90 & 0.206 \\
\hline & $51-60$ & $268(38.7)$ & 90 (33.6) & $88(32.8)$ & $90(33.6)$ & & \\
\hline & $>60$ & $238(34.4)$ & $76(31.9)$ & $91(38.2)$ & $71(29.8)$ & & \\
\hline \multirow[t]{3}{*}{ Diabetes Duration, year } & $<5$ & $341(50.8)$ & $111(32.6)$ & $106(31.1)$ & $124(36.4)$ & 8.61 & 0.072 \\
\hline & $5-9.9$ & $187(27.9)$ & $58(31.0)$ & $60(32.1)$ & 69 (36.9) & & \\
\hline & $\geq 10$ & $143(21.3)$ & $52(36.4)$ & 57 (39.9) & $34(23.8)$ & & \\
\hline \multirow[t]{2}{*}{ Gender } & Female & 365 (52.8) & $120(32.9)$ & $127(34.8)$ & $118(32.3)$ & 0.79 & 0.673 \\
\hline & Male & $326(47.2)$ & $106(32.5)$ & 105 (32.2) & 115 (35.3) & & \\
\hline \multirow[t]{3}{*}{ Ethnicity } & Malay & $365(53.1)$ & $109(29.9)$ & $120(32.9)$ & $136(37.3)$ & 16.48 & 0.002 \\
\hline & Chinese & $160(23.3)$ & 73 (45.6) & $46(28.8)$ & $41(25.6)$ & & \\
\hline & Indian & $163(23.7)$ & $46(28.2)$ & $62(38.0)$ & $55(33.7)$ & & \\
\hline \multirow[t]{6}{*}{ Religion } & No religion & $32(4.6)$ & $13(40.6)$ & $10(31.3)$ & $9(28.1)$ & 26.22 & 0.003 \\
\hline & Moslem & $373(53.9)$ & $109(29.2)$ & $124(33.2)$ & $140(37.5)$ & & \\
\hline & Buddhist & $81(11.7)$ & 45 (55.6) & 19 (23.5) & $17(21.0)$ & & \\
\hline & Hinduism/Sikh & $147(21.2)$ & 41 (27.9) & $57(38.8)$ & 49 (33.3) & & \\
\hline & Christian/Catholic & $22(3.2)$ & $7(31.8)$ & $7(31.8)$ & $8(36.4)$ & & \\
\hline & Others & $37(5.3)$ & $13(35.1)$ & $14(37.8)$ & $10(27.0)$ & & \\
\hline \multirow[t]{3}{*}{ Religiosity } & Religious & $590(85.5)$ & $183(31.0)$ & $197(33.4)$ & $210(35.6)$ & 11.95 & 0.018 \\
\hline & Unsure & $24(3.5)$ & $13(54.2)$ & $9(37.5)$ & $2(8.3)$ & & \\
\hline & Not religious & $76(11.0)$ & 31 (40.8) & 25 (32.9) & $20(26.3)$ & & \\
\hline \multirow[t]{4}{*}{ Marital status } & Married/living with a partner & $546(79.1)$ & $176(32.2)$ & $184(33.7)$ & $186(34.1)$ & 4.69 & 0.584 \\
\hline & Widowed & $97(14.1)$ & $30(30.9)$ & $35(36.1)$ & $32(33.0)$ & & \\
\hline & Divorced/separated & $21(3.0)$ & $11(52.4)$ & $4(19.0)$ & $6(28.6)$ & & \\
\hline & Single & $26(3.8)$ & $10(38.5)$ & $8(30.8)$ & $8(30.8)$ & & \\
\hline \multirow[t]{3}{*}{ Education } & Never & $45(6.6)$ & $18(40.0)$ & $16(35.6)$ & $11(24.4)$ & 7.15 & 0.128 \\
\hline & Primary \& secondary & $563(82.4)$ & $185(32.9)$ & $195(34.6)$ & $183(32.5)$ & & \\
\hline & Tertiary & $75(11.0)$ & $22(29.3)$ & $19(25.3)$ & $34(45.3)$ & & \\
\hline \multirow[t]{3}{*}{ Employment } & Retired/home manager & $368(53.3)$ & $127(34.5)$ & $131(35.6)$ & $110(29.9)$ & 7.64 & 0.106 \\
\hline & Employed & $312(45.2)$ & $95(30.4)$ & $98(31.4)$ & $119(38.1)$ & & \\
\hline & Unemployed & $11(1.6)$ & $6(54.5)$ & $2(18.2)$ & $3(27.3)$ & & \\
\hline \multirow[t]{3}{*}{ Income (RM) } & $<1000$ & $323(47.4)$ & $113(35.0)$ & $101(31.3)$ & $109(33.7)$ & 3.32 & 0.506 \\
\hline & 1000-2999 & $320(47.0)$ & 99 (30.9) & $115(35.9)$ & $106(33.1)$ & & \\
\hline & $\geq 3000$ & $38(5.6)$ & $9(23.7)$ & $14(36.8)$ & $15(39.5)$ & & \\
\hline \multirow[t]{3}{*}{ Exercise } & No & $293(42.5)$ & $112(38.2)$ & $91(31.1)$ & $90(30.7)$ & 10.94 & 0.027 \\
\hline & $\leq 3$ times in a week & $231(33.5)$ & 77 (33.3) & $79(34.2)$ & $75(32.5)$ & & \\
\hline & $>3$ times in a week & $166(24.1)$ & $39(23.5)$ & $60(36.1)$ & $67(40.4)$ & & \\
\hline \multirow[t]{3}{*}{ Smoking } & Never & $526(76.1)$ & $177(33.7)$ & $175(33.3)$ & $174(33.1)$ & 5.14 & 0.273 \\
\hline & Stop $>5$ years & $60(8.7)$ & $20(33.3)$ & $14(23.3)$ & $26(43.3)$ & & \\
\hline & Stop $\leq 5$ years and active smoker & 105 (15.2) & $32(30.5)$ & $41(39.0)$ & $32(30.5)$ & & \\
\hline
\end{tabular}


Table 1 The socio-demographic and clinical characteristics according to the tertile of the World Health Organization Quality of Life- Brief (WHOQOL-BREF) (Continued)

\begin{tabular}{|c|c|c|c|c|c|c|c|}
\hline \multirow[t]{3}{*}{$\overline{B M l}, \mathrm{Kgm}^{-2}$} & $<23.0$ & 76 (11.2) & $32(42.1)$ & $22(28.9)$ & $22(28.9)$ & 7.75 & 0.101 \\
\hline & $23.0-27.4$ & $237(35.0)$ & $82(34.6)$ & $82(34.6)$ & 73 (30.8) & & \\
\hline & $\geq 27.5$ & 365 (53.8) & $103(28.1)$ & $125(34.2)$ & $137(37.5)$ & & \\
\hline \multirow[t]{4}{*}{$\mathrm{HbA1c}, \%$} & $\mathrm{HbA1c} \geq 6.5$ & $515(83.3)$ & 168 (32.6) & $171(33.2)$ & $176(34.2)$ & 0.26 & 0.879 \\
\hline & $\mathrm{HbA} 1 \mathrm{c}<6.5$ & $103(16.7)$ & $36(35.0)$ & $34(33.0)$ & $33(32.0)$ & & \\
\hline & $\mathrm{HbA} 1 \mathrm{c} \geq 7.0$ & 455 (73.6) & $148(32.5)$ & 153 (33.6) & 154 (33.8) & 0.23 & 0.892 \\
\hline & $\mathrm{HbA} 1 \mathrm{c}<7.0$ & $163(26.4)$ & $56(34.4)$ & $52(31.9)$ & $55(33.7)$ & & \\
\hline \multirow[t]{2}{*}{ Hypertension } & No & $148(21.7)$ & $46(31.1)$ & $45(30.4)$ & $57(38.5)$ & 1.71 & 0.426 \\
\hline & Yes & $534(78.3)$ & $180(33.7)$ & $179(33.5)$ & 175 (32.8) & & \\
\hline \multirow[t]{2}{*}{$\mathrm{BP}, \mathrm{mmHg}$} & $B P>130 / 80$ & 479 (69.5) & 155 (32.4) & $155(32.4)$ & 169 (35.3) & 1.53 & 0.466 \\
\hline & $B P \leq 130 / 80$ & $210(30.5)$ & $72(34.3)$ & $74(35.2)$ & $64(30.5)$ & & \\
\hline \multirow[t]{2}{*}{ Dyslipidaemia } & No & $406(60.9)$ & $124(30.5)$ & 125 (30.8) & $157(38.7)$ & 9.94 & 0.007 \\
\hline & Yes & 261 (39.1) & $95(36.4)$ & 96 (36.8) & $70(26.8)$ & & \\
\hline \multirow[t]{2}{*}{$\mathrm{LDL}-\mathrm{C}, \mathrm{mmol} / \mathrm{L}$} & $\mathrm{LDL}-\mathrm{C}>2.6$ & $336(59.9)$ & $106(31.5)$ & $120(35.7)$ & $110(32.7)$ & 2.72 & 0.257 \\
\hline & $\mathrm{LDL}-\mathrm{C} \leq 2.6$ & $225(40.1)$ & 74 (32.9) & $66(29.3)$ & 85 (37.8) & & \\
\hline \multirow[t]{2}{*}{$\mathrm{HDL}-\mathrm{C}, \mathrm{mmol} / \mathrm{L}$} & $\mathrm{HDL}-\mathrm{C}<1.1$ & $407(72.2)$ & $118(29.0)$ & $143(35.1)$ & 146 (35.9) & 5.86 & 0.053 \\
\hline & $\mathrm{HDL}-\mathrm{C} \geq 1.1$ & $157(27.8)$ & $62(39.5)$ & 45 (28.7) & $50(31.8)$ & & \\
\hline \multirow[t]{2}{*}{$\mathrm{TG}, \mathrm{mmol} / \mathrm{L}$} & $\mathrm{TG}>1.7$ & $250(44.3)$ & $81(32.4)$ & $85(34.0)$ & 84 (33.6) & 0.36 & 0.837 \\
\hline & $\mathrm{TG} \leq 1.7$ & $314(55.7)$ & 99 (31.5) & $102(32.5)$ & $113(36.0)$ & & \\
\hline \multirow[t]{2}{*}{ Total-C, mmol/L } & Total-C $>4.5$ & $357(58.1)$ & 121 (33.9) & $126(35.3)$ & $110(30.8)$ & 4.81 & 0.090 \\
\hline & Total-C $\leq 4.5$ & $257(41.9)$ & 78 (30.4) & $78(30.4)$ & $101(39.3)$ & & \\
\hline \multirow[t]{2}{*}{ Any diabetes complication } & No & $608(87.7)$ & $193(31.7)$ & $201(33.1)$ & $214(35.2)$ & 6.31 & 0.043 \\
\hline & Yes & $85(12.3)$ & $36(42.4)$ & $30(35.3)$ & $19(22.4)$ & & \\
\hline \multirow[t]{2}{*}{ Microvascular complication } & No & $641(92.5)$ & $207(32.3)$ & $212(33.1)$ & $222(34.6)$ & 4.23 & 0.120 \\
\hline & Yes & $52(7.5)$ & $22(42.3)$ & $19(36.5)$ & $11(21.2)$ & & \\
\hline \multirow[t]{2}{*}{ Macrovascular complication } & No & $648(93.9)$ & $206(31.8)$ & $217(33.5)$ & $225(34.7)$ & 6.92 & 0.031 \\
\hline & Yes & $42(6.1)$ & $21(50.0)$ & $13(31.0)$ & $8(19.0)$ & & \\
\hline \multirow[t]{2}{*}{$\mathrm{OHA}$} & No & $60(8.7)$ & $25(41.7)$ & $20(33.3)$ & $15(25.0)$ & 3.03 & 0.220 \\
\hline & Yes & $630(91.3)$ & $202(32.1)$ & $210(33.3)$ & $218(34.6)$ & & \\
\hline \multirow[t]{3}{*}{ Insulin } & No & $422(61.2)$ & $132(31.3)$ & $139(32.9)$ & $151(35.8)$ & 2.27 & 0.687 \\
\hline & 1 type & $187(27.1)$ & $66(35.3)$ & $64(34.2)$ & $57(30.5)$ & & \\
\hline & 2 types & $80(11.6)$ & $29(36.3)$ & $26(32.5)$ & $25(31.3)$ & & \\
\hline \multirow[t]{4}{*}{ Number of AHA agents } & No & $81(11.8)$ & $22(27.2)$ & $30(37.0)$ & $29(35.8)$ & 4.71 & 0.581 \\
\hline & 1 type & 204 (29.6) & 70 (34.3) & 65 (31.9) & 69 (33.8) & & \\
\hline & 2 types & $203(29.5)$ & $73(36.0)$ & $59(29.1)$ & $71(35.0)$ & & \\
\hline & 3 types & $201(29.2)$ & $62(30.8)$ & 75 (37.3) & $64(31.8)$ & & \\
\hline \multirow[t]{2}{*}{ LLA } & No & $156(22.6)$ & $50(32.1)$ & $47(30.1)$ & $59(37.8)$ & 1.59 & 0.451 \\
\hline & Yes & $533(77.4)$ & 177 (33.2) & $182(34.1)$ & 174 (32.6) & & \\
\hline \multirow[t]{2}{*}{ APA } & No & $612(89.1)$ & $194(31.7)$ & $202(33.0)$ & $216(35.3)$ & 6.48 & 0.039 \\
\hline & Yes & 75 (10.9) & $32(42.7)$ & $27(36.0)$ & $16(21.3)$ & & \\
\hline
\end{tabular}

\footnotetext{
${ }^{*}$ Chi-square $P$ value
}

$B P$ blood pressure, LDL-C low-density lipoprotein cholesterol, HDL-C high-density lipoprotein cholesterol, TG triglycerides, Total-C total cholesterol, AHA anti-

hypertensive agent, $B M I$ body mass index, $B P$ blood pressure, $A P A$ anti-platelet agent, $O H A$ oral hypoglycaemic agent, $L D L-C$ low density lipoprotein- cholesterol,

HDL-C high density lipoprotein- cholesterol, LLA lipid-lowering agent, RM Ringgit Malaysia, TG triglyceride, Total-C total-cholesterol, WHOQoL-BREF World Health

Organization Quality of Life-Brief 26 items

${ }^{a}$ Column percentage

${ }^{b}$ Row percentage 


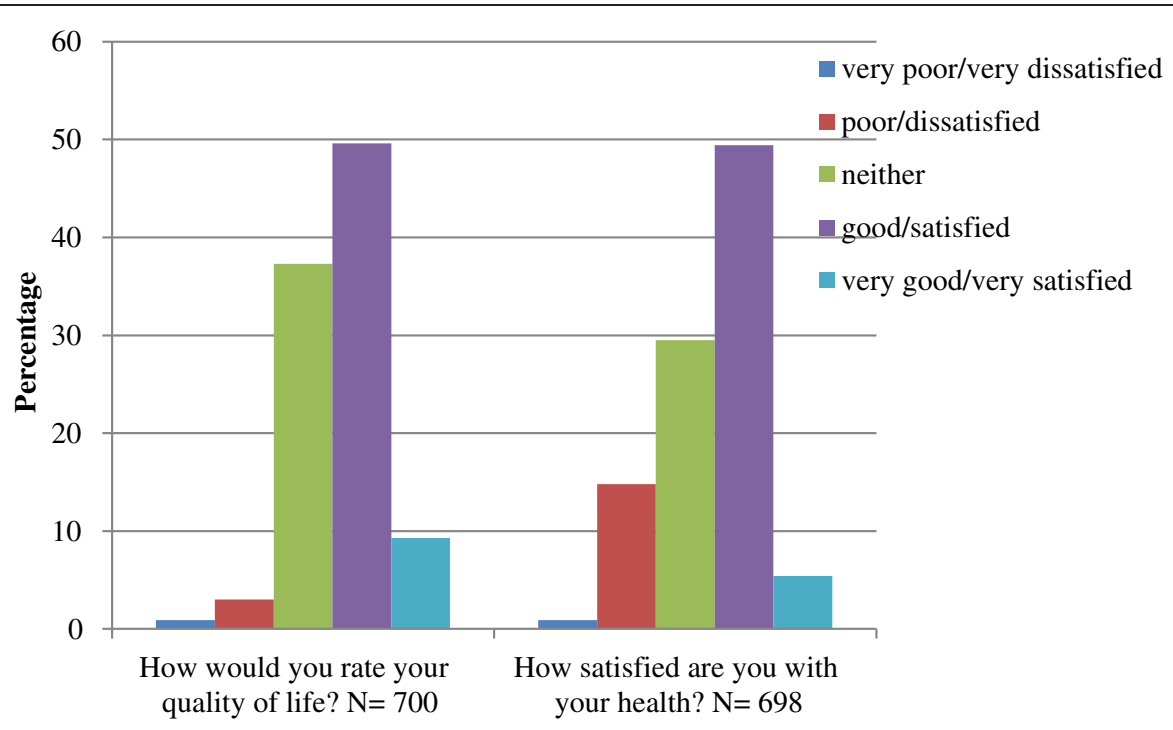

Fig. 1 The proportion of responses to the first two items of the WHOQOL-BREF on the overall perception of health. WHOQOL-BREF= World Health Organization Quality of Life- Brief

$(\mathrm{B}=-4.51)$ and Total- $\mathrm{C}$ on EQOL $(\mathrm{B}=-1.04)$. Patients who were on OHA compared to those not taking any OHA were reported to have better HRQoL, and the most significant positive effect was seen on PQOL (Table 2).

\section{Diabetes-related distress, depressive symptoms and quality of life}

The mean (SD) for total DDS-17 score was 37.1 (15.98) with $19.6 \%(136 / 694)$ had moderate distress. Both higher DRD and DS were associated with lower HRQoL,

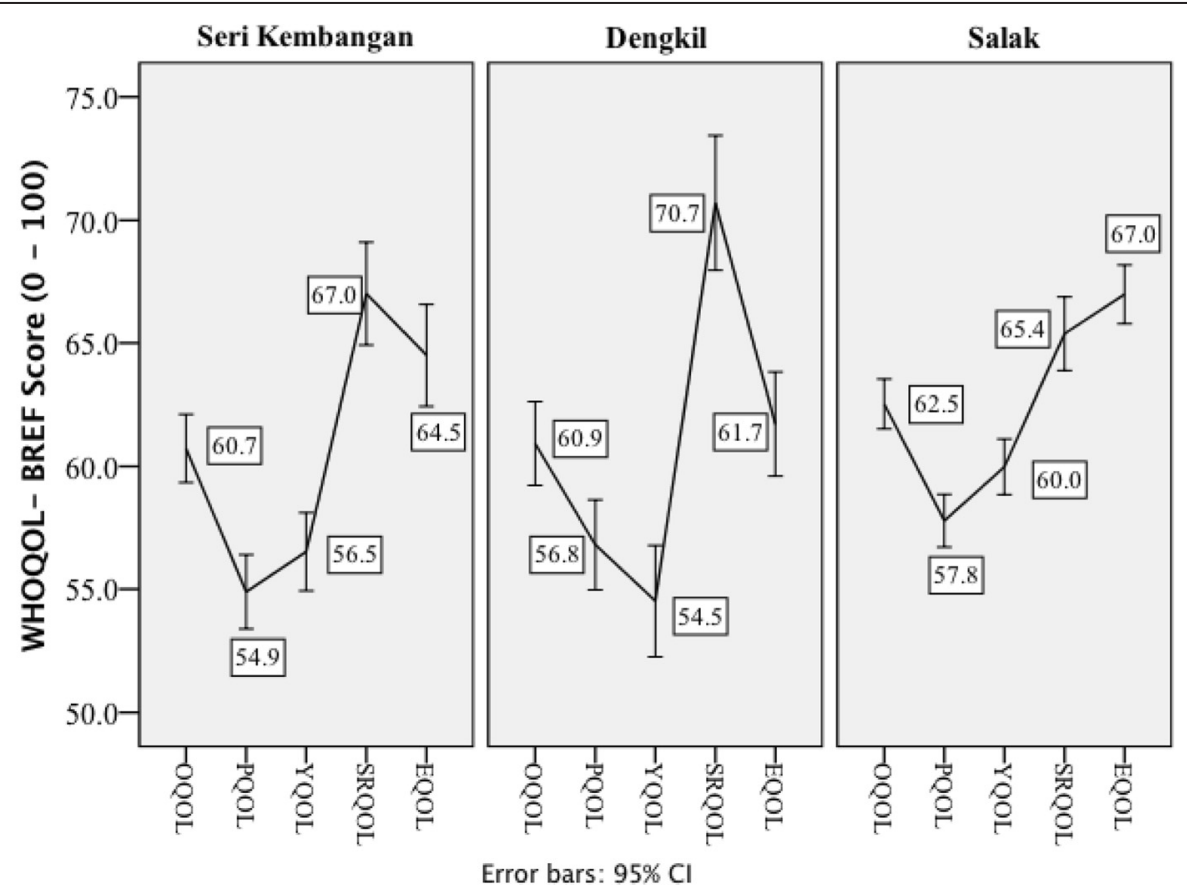

WHOQoL-BREF $=$ World Health Organization Quality of Life-Brief 26 items $\mathrm{OQOL}=$ overall quality of life, $\mathrm{PQOL}=$ Physical quality of life, $\mathrm{YQOL}=$ Psychological quality of life, $\mathrm{SRQOL}=$ Social Relationships quality of life, EQOL= Environment quality of life

Fig. 2 The WHOQOL-BREF mean (95\% confidence interval) of the total and domain score according to the three primary healthcare clinics in Malaysia 
Table 2 The determinants of the health-related quality of life measured by the World Health Organization Quality of Life- Brief (WHOQOL-BREF)

\begin{tabular}{|c|c|c|c|c|c|c|c|c|c|c|}
\hline & \multicolumn{2}{|l|}{ OQOL, $n=490$} & \multicolumn{2}{|l|}{$\mathrm{PQOL}, n=472$} & \multicolumn{2}{|l|}{ YQOL, $n=457$} & \multicolumn{2}{|l|}{ SRQOL, $n=560$} & \multicolumn{2}{|l|}{ EQOL, $n=535$} \\
\hline & $\begin{array}{l}\text { Crude B } \\
(95.0 \% \mathrm{Cl})\end{array}$ & $\begin{array}{l}\text { Adjusted B } \\
(95.0 \% \mathrm{Cl})\end{array}$ & $\begin{array}{l}\text { Crude B } \\
(95.0 \% \text { Cl) }\end{array}$ & $\begin{array}{l}\text { Adjusted B } \\
(95.0 \% \mathrm{Cl})\end{array}$ & $\begin{array}{l}\text { Crude B } \\
(95.0 \% \text { Cl) }\end{array}$ & $\begin{array}{l}\text { Adjusted B } \\
(95.0 \% \mathrm{Cl})\end{array}$ & $\begin{array}{l}\text { Crude B } \\
(95.0 \% \text { Cl) }\end{array}$ & $\begin{array}{l}\text { Adjusted B } \\
(95.0 \% \mathrm{Cl})\end{array}$ & $\begin{array}{l}\text { Crude B } \\
(95.0 \% \text { Cl) }\end{array}$ & $\begin{array}{l}\text { Adjusted B } \\
(95.0 \% \mathrm{Cl})\end{array}$ \\
\hline (Constant) & - & $\begin{array}{l}71.36 \\
(61.34,81.38)\end{array}$ & - & $\begin{array}{l}60.18 \\
(48.9,71.44)\end{array}$ & - & $\begin{array}{l}69.38 \\
(55.35,83.41)\end{array}$ & - & $\begin{array}{l}76.54 \\
(65.10,87.99)\end{array}$ & - & $\begin{array}{l}69.34 \\
(54.07,81.61)\end{array}$ \\
\hline DDS score ${ }^{a}$ & $\begin{array}{l}-0.18 \\
(-0.22,-0.13)\end{array}$ & $\begin{array}{l}-0.04 \\
(-0.10,0.02)\end{array}$ & $\begin{array}{l}-0.13 \\
(-0.17,-0.08)\end{array}$ & $\begin{array}{l}0.01 \\
(-0.06,0.08)\end{array}$ & $\begin{array}{l}-0.18 \\
(-0.23,-0.13)\end{array}$ & $\begin{array}{l}-0.12 \\
(-0.20,-0.04)\end{array}$ & $\begin{array}{l}-0.19 \\
(-0.26,-0.12)\end{array}$ & $\begin{array}{l}0.001 \\
(-0.09,0.09)\end{array}$ & $\begin{array}{l}-0.22 \\
(-0.28,-0.16)\end{array}$ & $\begin{array}{l}-0.07 \\
(-0.14,0.01)\end{array}$ \\
\hline PHQ score ${ }^{b}$ & $\begin{array}{l}-0.95 \\
(-1.11,-0.80)\end{array}$ & $\begin{array}{l}-0.81 \\
(-1.02,-0.60)\end{array}$ & $\begin{array}{l}-0.95 \\
(-1.12,-0.77)\end{array}$ & $\begin{array}{l}-0.87 \\
(-1.11,-0.63)\end{array}$ & $\begin{array}{l}-0.68 \\
(-0.87,-0.48)\end{array}$ & $\begin{array}{l}-0.42 \\
(-0.69,-0.14)\end{array}$ & $\begin{array}{l}-1.19 \\
(-1.43,-0.95)\end{array}$ & $\begin{array}{l}-1.21 \\
(-1.52,-0.90)\end{array}$ & $\begin{array}{l}-1.02 \\
(-1.23,-0.80)\end{array}$ & $\begin{array}{l}-0.74 \\
(-1.02,-0.47)\end{array}$ \\
\hline Men & $\begin{array}{l}0.36 \\
(-1.12,1.83)\end{array}$ & $\begin{array}{l}-0.76 \\
(-2.42,0.90)\end{array}$ & $\begin{array}{l}1.70 \\
(0.12,3.29)\end{array}$ & $\begin{array}{l}0.21 \\
(-1.73,2.14)\end{array}$ & $\begin{array}{l}0.75 \\
(-1.01,2.50)\end{array}$ & $\begin{array}{l}-0.19 \\
(-2.42,2.05)\end{array}$ & $\begin{array}{l}-1.86 \\
(-4.10,0.38)\end{array}$ & $\begin{array}{l}-2.70 \\
(-5.06,-0.35)\end{array}$ & $\begin{array}{l}0.90 \\
(-1.05,2.84)\end{array}$ & $\begin{array}{l}-0.06 \\
(-2.16,2.03)\end{array}$ \\
\hline Malay ${ }^{d}$ & $\begin{array}{l}1.94 \\
(0.47,3.42)\end{array}$ & $\begin{array}{l}0.67 \\
(-1.11,2.45)\end{array}$ & $\begin{array}{l}1.35 \\
(-0.24,2.94)\end{array}$ & $\begin{array}{l}-0.70 \\
(-2.78,1.37)\end{array}$ & $\begin{array}{l}2.58 \\
(0.83,4.33)\end{array}$ & $\begin{array}{l}-0.57 \\
(-2.96,1.82)\end{array}$ & $\begin{array}{l}2.58 \\
(0.83,4.33)\end{array}$ & $\begin{array}{l}1.21 \\
(-1.15,3.56)\end{array}$ & $\begin{array}{l}2.81 \\
(0.87,4.75)\end{array}$ & $\begin{array}{l}2.60 \\
(0.33,4.88)\end{array}$ \\
\hline Age (year) & $\begin{array}{l}-0.06 \\
(-0.13,0.02)\end{array}$ & $\begin{array}{l}-0.06 \\
(-0.15,0.03)\end{array}$ & $\begin{array}{l}-0.14 \\
(-0.21,-0.06)\end{array}$ & $\begin{array}{l}-0.06 \\
(-0.16,0.05)\end{array}$ & $\begin{array}{l}-0.14 \\
(-0.23,-0.06)\end{array}$ & $\begin{array}{l}-0.11 \\
(-0.24,0.01)\end{array}$ & $\begin{array}{l}0.01 \\
(-0.10,0.12)\end{array}$ & $\begin{array}{l}-0.12 \\
(-0.24,-0.003)\end{array}$ & $\begin{array}{l}0.05 \\
(-0.05,0.14)\end{array}$ & $\begin{array}{l}-0.01 \\
(-0.11,0.10)\end{array}$ \\
\hline $\begin{array}{l}\text { Diabetes } \\
\text { duration (year) }\end{array}$ & - & - & $\begin{array}{l}-0.18 \\
(-0.32,-0.04)\end{array}$ & $\begin{array}{l}0.04 \\
(-0.13,0.21)\end{array}$ & $\begin{array}{l}-0.18 \\
(-0.34,-0.02)\end{array}$ & $\begin{array}{l}-0.03 \\
(-0.24,0.17)\end{array}$ & - & - & - & - \\
\hline Health clinic ${ }^{c}$ & $\begin{array}{l}0.93 \\
(0.10,1.76)\end{array}$ & $\begin{array}{l}-0.95 \\
(-2.32,0.425)\end{array}$ & $\begin{array}{l}1.44 \\
(0.56,2.32)\end{array}$ & $\begin{array}{l}-0.46 \\
(-2.02,1.10)\end{array}$ & $\begin{array}{l}1.95 \\
(0.98,2.93)\end{array}$ & $\begin{array}{l}-0.78 \\
(-2.56,1.00)\end{array}$ & - & - & $\begin{array}{l}1.41 \\
(0.32,2.50)\end{array}$ & $\begin{array}{l}-1.00 \\
(-2.64,0.64)\end{array}$ \\
\hline Moslem ${ }^{e}$ & - & - & - & - & $\begin{array}{l}-4.85 \\
(-8.95,-0.75)\end{array}$ & $\begin{array}{l}0.48 \\
(-5.52,6.32)\end{array}$ & - & - & - & - \\
\hline Religiosity $^{f}$ & $\begin{array}{l}3.93 \\
(1.86,6.00)\end{array}$ & $\begin{array}{l}3.07 \\
(0.46,5.67)\end{array}$ & $\begin{array}{l}-2.37 \\
(-3.60,-1.14)\end{array}$ & $\begin{array}{l}3.22 \\
(0.27,6.18)\end{array}$ & $\begin{array}{l}5.29 \\
(2.85,7.73)\end{array}$ & $\begin{array}{l}4.76 \\
(1.15,8.36)\end{array}$ & - & - & - & - \\
\hline Not married & - & - & - & - & $\begin{array}{l}-2.20 \\
(-4.35,-0.06)\end{array}$ & $\begin{array}{l}-2.11 \\
(-4.62,0.40)\end{array}$ & - & - & $\begin{array}{l}-2.54 \\
(-4.94,-0.15)\end{array}$ & $\begin{array}{l}-2.82 \\
(-5.39,-0.25)\end{array}$ \\
\hline $\begin{array}{l}\text { Educational } \\
\text { status }^{9}\end{array}$ & - & - & $\begin{array}{l}3.25 \\
(1.36,5.14)\end{array}$ & $\begin{array}{l}0.88 \\
(-1.42,3.17)\end{array}$ & $\begin{array}{l}2.86 \\
(0.76,4.96)\end{array}$ & $\begin{array}{l}0.76 \\
(-1.83,3.34)\end{array}$ & - & - & - & - \\
\hline $\begin{array}{l}\text { Employment } \\
\text { statush }\end{array}$ & $\begin{array}{l}1.41 \\
(0.03,2.80)\end{array}$ & $\begin{array}{l}2.08 \\
(0.43,3.72)\end{array}$ & $\begin{array}{l}2.93 \\
(1.46,4.41)\end{array}$ & $\begin{array}{l}2.34 \\
(0.46,4.22)\end{array}$ & $\begin{array}{l}2.59 \\
(0.93,4.24)\end{array}$ & $\begin{array}{l}1.31 \\
(-0.83,3.45)\end{array}$ & - & - & - & - \\
\hline Exercise $e^{i}$ & $\begin{array}{l}1.61 \\
(0.69,2.53)\end{array}$ & $\begin{array}{l}1.31 \\
(0.32,2.29)\end{array}$ & $\begin{array}{l}1.01 \\
(0.01,2.00)\end{array}$ & $\begin{array}{l}1.08 \\
(-0.04,2.20)\end{array}$ & - & - & $\begin{array}{l}2.61 \\
(1.21,4.00)\end{array}$ & $\begin{array}{l}2.38 \\
(0.93,3.83)\end{array}$ & $\begin{array}{l}2.05 \\
(0.84,3.26)\end{array}$ & $\begin{array}{l}1.99 \\
(0.69,3.29)\end{array}$ \\
\hline BMI $\left(\mathrm{kgm}^{-2}\right)$ & - & - & - & - & $\begin{array}{l}0.16 \\
(0.0001,0.31)\end{array}$ & $\begin{array}{l}0.08 \\
(-0.14,0.29)\end{array}$ & - & - & - & - \\
\hline SBP $(\mathrm{mmHg})$ & - & - & - & - & - & - & - & - & $\begin{array}{l}0.07 \\
(0.01,0.12)\end{array}$ & $\begin{array}{l}0.07 \\
(0.01,0.13)\end{array}$ \\
\hline $\begin{array}{l}\mathrm{BP}<130 / 80 \\
\mathrm{mmHg}\end{array}$ & - & - & $\begin{array}{l}-1.11 \\
(-2.10,-0.13)\end{array}$ & $\begin{array}{l}-1.43 \\
(-3.37 .0 .51)\end{array}$ & - & - & - & - & - & - \\
\hline $\begin{array}{l}\text { Hypertension } \\
\text { (Yes) }\end{array}$ & - & - & $\begin{array}{l}-2.44 \\
(-4.37,-0.50)\end{array}$ & $\begin{array}{l}-1.73 \\
(-4.05,0.58)\end{array}$ & $\begin{array}{l}-2.83 \\
(-4.95,-0.71)\end{array}$ & $\begin{array}{l}-1.97 \\
(-4.58,0.63)\end{array}$ & $\begin{array}{l}2.96 \\
(0.22,5.70)\end{array}$ & $\begin{array}{l}2.57 \\
(-0.29,5.42)\end{array}$ & - & - \\
\hline
\end{tabular}


Table 2 The determinants of the health-related quality of life measured by the World Health Organization Quality of Life- Brief (WHOQOL-BREF) (Continued)

\begin{tabular}{|c|c|c|c|c|c|c|c|c|c|c|}
\hline CBG (mmol/L) & $\begin{array}{l}-0.30 \\
(-0.50,-0.10)\end{array}$ & $\begin{array}{l}-0.22 \\
(-0.44,0.001)\end{array}$ & - & - & - & - & - & - & - & - \\
\hline $\mathrm{HDL}-\mathrm{C}(\mathrm{mmol} / \mathrm{L})$ & $\begin{array}{l}-3.82 \\
(-6.34,-1.31)\end{array}$ & $\begin{array}{l}-2.71 \\
(-5.49,0.06)\end{array}$ & $\begin{array}{l}-7.17 \\
(-9.86,-4.48)\end{array}$ & $\begin{array}{l}-4.51 \\
(-7.58,-1.44)\end{array}$ & $\begin{array}{l}-3.96 \\
(-6.94,-0.99)\end{array}$ & $\begin{array}{l}-1.42 \\
(-4.91,2.07)\end{array}$ & - & - & - & - \\
\hline Total-C (mmol/L) & $\begin{array}{l}-0.72 \\
(-1.40,-0.05)\end{array}$ & $\begin{array}{l}-0.19 \\
(-0.91,0.54)\end{array}$ & - & - & - & - & $\begin{array}{l}-1.09 \\
(-2.11,-0.08)\end{array}$ & $\begin{array}{l}-0.63 \\
(-1.65,0.40)\end{array}$ & $\begin{array}{l}-0.90 \\
(-1.80,-0.01)\end{array}$ & $\begin{array}{l}-1.04 \\
(-1.94,-0.15)\end{array}$ \\
\hline $\begin{array}{l}\text { Dyslipidaemia } \\
\text { (Yes) }\end{array}$ & $\begin{array}{l}-2.18 \\
(-3.71,-0.65)\end{array}$ & $\begin{array}{l}-1.53 \\
(-3.52,0.46)\end{array}$ & $\begin{array}{l}-1.98 \\
(-3.61,-0.34)\end{array}$ & $\begin{array}{l}0.32 \\
(-1.96,2.59)\end{array}$ & $\begin{array}{l}-4.75 \\
(-6.54,-2.96)\end{array}$ & $\begin{array}{l}-3.14 \\
(-5.78,-0.50)\end{array}$ & - & - & $\begin{array}{l}-3.76 \\
(-5.75,-1.76)\end{array}$ & $\begin{array}{l}-2.78 \\
(-4.48,-0.07)\end{array}$ \\
\hline MicroCx (Yes) & - & - & - & - & $\begin{array}{l}-3.28 \\
(-6.66,0.003)\end{array}$ & $\begin{array}{l}-0.07 \\
(-2.78,2.64)\end{array}$ & - & - & $\begin{array}{l}-4.34 \\
(-7.99,-0.70)\end{array}$ & $\begin{array}{l}-1.40 \\
(-4.28,1.48)\end{array}$ \\
\hline MacroCx (Yes) & $\begin{array}{l}-4.31 \\
(-7.38,-1.23)\end{array}$ & $\begin{array}{l}-4.98 \\
(-8.56,-1.40)\end{array}$ & $\begin{array}{l}-4.45 \\
(-7.77,-1.13)\end{array}$ & $\begin{array}{l}-3.50 \\
(-7.36,0.37)\end{array}$ & $\begin{array}{l}-4.80 \\
(-8.45,-1.14)\end{array}$ & $\begin{array}{l}-4.45 \\
(-9.42,0.52)\end{array}$ & - & - & $\begin{array}{l}-4.44 \\
(-8.51,-0.38)\end{array}$ & $\begin{array}{l}-4.38 \\
(-8.30,-0.46)\end{array}$ \\
\hline OHA (Yes) & $\begin{array}{l}3.02 \\
(0.41,5.63)\end{array}$ & $\begin{array}{l}1.56 \\
(-1.40,4.51)\end{array}$ & $\begin{array}{l}3.42 \\
(0.62,6.21)\end{array}$ & $\begin{array}{l}3.82 \\
(0.43,7.21)\end{array}$ & - & - & $\begin{array}{l}5.37 \\
(1.42,9.31)\end{array}$ & $\begin{array}{l}3.01 \\
(-1.24,7.26)\end{array}$ & - & - \\
\hline APA (Yes) & $\begin{array}{l}-2.73 \\
(-5.10,-0.37)\end{array}$ & $\begin{array}{l}0.13 \\
(-2.56,2.81)\end{array}$ & $\begin{array}{l}-3.35 \\
(-5.89,-0.81)\end{array}$ & $\begin{array}{l}-1.53 \\
(-4.65,1.59)\end{array}$ & $\begin{array}{l}-5.28 \\
(-8.08,-2.49)\end{array}$ & $\begin{array}{l}-0.15 \\
(-3.73,3.42)\end{array}$ & - & - & - & - \\
\hline
\end{tabular}

Bs are unstandardized coefficients

$O Q O L$ overall quality of life, $P Q O L$ physical quality of life, YQOL psychological quality of life, SRQOL social relationships quality of life, EQOL environment quality of life, $A H A$ anti-hypertensive agent, APA anti-platelet agent, $B$ Coefficients, $C B G$ casual blood glucose, $B M I$ body mass index, $C l$ confidence interval, DDS diabetes distress scale, MacroCx macrovascular complication, Micro $C x$ microvascular complication, OHA oral hypoglycaemic agent, $P H Q$ patient health questionnaire, SBP systolic blood pressure, Total-C total cholesterol

aTotal DDS scores range from 17 to 102
${ }^{\text {b}}$ Total PHQ-9 scores range from 0 to 27

'Health clinic = Seri Kembangan (1), Dengkil (2), Salak (3)

${ }^{\mathrm{d} N o n-M a l a y ~ a s ~ r e f e r e n c e ~}$

eNon-Moslem as reference

Non-religious as reference

${ }^{9}$ Educational status = never (0), primary \& secondary (1), tertiary and above (2)

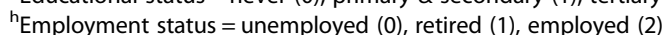

${ }^{i}$ Exercise $=$ no $(0)$, at most three times per week (1), more than three times per week (2) 
especially on the YQOL (Table 2). DS showed more consistent, negative and independent effects across all the domains of HRQoL in comparison with DRD.

\section{Discussion}

This study examined the HRQoL and its determinants in adults with T2D at the primary care level. WHOQOLBREF was used instead of the disease-specific measure in order to determine the wider life impacts of T2D on patients which was rarely studied in the past. Nevertheless, in addition to the DS assessment a disease specific measure for distress, the DDS-17, was included in the present study.

It was disturbing to find that the mean score for PQOL was the lowest followed by YQOL. PQOL which represented physical functions, disability and needs showed that adult patients with $\mathrm{T} 2 \mathrm{D}$ required much care for their pain, sleep and mobility. They also lacked of support from others to satisfactorily perform their daily activities. Physical problems such as pain, discomfort, and diet restrictions were also known to cause major problems in adult patients with T2D who were treated at primary care level in Singapore [50]. Perhaps more practical and psychological supports are needed to alleviate their perception on the burden of taking medication in order to improve their confidence when performing house chores and working. The findings on the YQOL domain revealed that adult patients with T2D experienced negative feelings (blue moods, despair, anxiety etc.) at primary care level which resulted in the inability to concentrate or enjoy a meaningful life. These two domains of poor physical and mental health had also been reported to have caused a median of 2 (IQR 0-10) unhealthy days in the past month among Irish patients with diabetes mellitus [51].

The SRQOL was the least affected among all the WHOQOL-BREF domains, and this was probably due to the prevalent of social support [52], culture [53], and family values such as filial piety in this country and region $[54,55]$. However, these findings and values are not restricted to this study and region [25]. The perception on the quality of environment, living conditions, and access to transport and healthcare was categorised as moderate to good, and this was probably due to moderate ways of living and the culture of accepting current situations or fates. Although these personal attitudes and virtues could pose real barriers to diabetes care [56], it was uncertain whether having these qualities would help the patients to face life challenges and contribute to the high (about one third) responses of neither good nor dissatisfied in the first two items of WHOQOL-BREF.

\section{Socio-demographic profile and quality of life}

Men with T2D were generally experienced lower HRQoL and significantly so in SRQOL except in PQOL. It was possible that these men were almost inherently the bread-winners of their families and were required to be out-and-about, and were less likely to complain or perceive themselves to be in need of basic help (just like men in most cultures). Hence, there was no association between these men and the physical domain of HRQoL [57]. In relation to men's emotional ability [58], it was noted that they experienced the poorest SRQOL that required emotional skills for interpersonal relationships and the management of emotions. These findings were in contrast to the men with T2D from multi-ethnic backgrounds (non-Hispanic whites, African-Americans, Asian-Indians, and Hispanics) in Texas, United States (US) who were reported to have better diabetes-specific HRQoL compared to the women [55]. In another study, women who perceived living with diabetes as predominantly stressful intermingled with depressive feelings were required to be constantly vigilant about healthy eating, self-concern, and fatigue [3]. In this study, it was noted that HRQoL was better perceived by women because T2D had more adverse impacts on men, or the men's perception could be limited by their emotional ability [58]. However, future studies are needed to confirm this and to examine the causes of low HRQoL among men with T2D.

Compared to other ethnic groups, Malay patients had a significantly better perception of their HRQoL in the univariable analyses. It was only statistically significant in the EQOL after the other covariates were adjusted and was negatively associated with the PQOL and YQOL domains (not reaching statistical significance). It was believed that this is closer to the actual states found in adults with T2D compared to the previous studies that reported positive association between Malays and their emotional and mental health status [59-61]. These past studies were limited by inadequate adjustments [59], loss of data quality due to the heterogeneity of the population (not specific to T2D), categorization of continuous outcome data and data-driven analysis $[60,61]$. The Malays experienced better HRQoL, and this could be due to being more religious and having better social support network when faced with the external challenges of living with T2D [53, 60]. Nevertheless, self-care is patently a personal issue, and the impacts of changed life routines due to T2D are irrespective of ethnicity as evidenced by the non-significant differential effects of ethnicity on many HRQoL domains in the multivariable analyses.

After adjusting for co-morbidities and complications, this study showed the negative impacts of older age and having diabetes for a longer duration on HRQoL, especially on the social relationship domain. In a study involving Greek respondents, a generic SF-36 instrument was used on T2D patients in a rural setting, and it was 
discovered that women who were older, had diabetes for a longer duration and were unmarried were the predictors of impaired HRQoL [32]. Similar results were also reported from a Turkish setting where diabetes-specific HRQoL assessment tool was used (DQoL) [62], and T2D patients who were older than 40 year old, men, unmarried, had diabetes for more than 5 years, suffered from complications or prior hospitalization, and had HbA1c $>7 \%$, a significantly poorer overall HRQoL. It was possible that T2D patients who were older or had diabetes for a longer duration had higher cardiovascular risk, were prescribed more medications (and insulin), had more scheduled visits to different medical specialists, suffered from more co-morbidities, and complications which had reduced HRQoL [63]. It was unfortunate that although social care and support were available [25], the older patients found themselves inadequate for acceptable quality of life.

Amongst all the socio-demographic variables, being a religious person was noted to be the most potent determinant for HRQoL. The salutogenic effects of religiosity could be linked to the privileged relationship with the Higher or Supreme Being, stronger spirituality, and having a sense of purpose in life, social network and healthy lifestyles [64-66]. The ability to adhere to one's religion and its way of life is defined as religiosity in this study. A patient must have had some physical agility that enabled him/her to attend places of worship and to participate in ceremonies or rites. Success in fulfilling these religious requirements might greatly ease any sense of guilt and improve spiritual well-being that could further induce a sense of harmony with the Supreme Being and psychological wellness $[67,68]$, which indeed is an internal resource for the self-management of T2D $[69,70]$. Spirituality and spiritual support were the constructs that could relate to DS and affect HRQoL in T2D patients [71-73]. However, more research is needed to understand the independent associations of religiosity and the differential effects of the different religions, on HRQoL.

Also, it was reassuring to find that being employed and engaged in high frequency exercise per week contributed to better HRQoL. Most likely active employment provided economic security and social status, and the absence of either one or both factors would be detrimental to many aspects of human living and quality of life. Since active employment was generally equated to higher socio-economic status and education, the effects of these factors on HRQoL were also reported elsewhere $[30,32,33]$. In a more optimistic note, patients who were in active vocations might feel more fulfilled when completing their jobs or attending job-related engagements which could be lacking if they were in the retirement phase.
Exercise and HRQoL have a multi-level and multidimensional association, and the effects are beneficial to patients with T2D [74]. In a nationwide survey in the US, it was observed that a self-reported exercise was the only significant self-management behaviour to predict HRQoL after controlling the demographic and medical variables [26]. Physiologically, exercise or physical activity stimulates the release of endorphin that induces and facilitates a sense of elation, relaxation, and well-being [75]. Psychologically, when T2D patients adhere closely to the advice to exercise regularly, this may encourage a sense of compliance and harmonious relationship with their healthcare professionals and significant others [9]. In return, regular and increased frequency of exercise could also increase the social connectivity between the patients, their family members and friends, and also improve their physical health [76].

\section{Clinical parameters and quality of life}

The negative and independent effects of MacroCx on OQOL were not unexpected because the consequences of IHD and stroke on patients' daily life activities are known to many [29, 77]. In contrast, the more subtle and minimal the impacts of the early stages of MicroCx would explain the lack of its effects on HRQoL. Similar findings were also reported with diabetes patients at primary care from the Nordic countries [28], Greece [32] and Malaysia [61]. Although many different QoL measures were used in these studies, the results showed consistency and strong negative effects of MacroCx, especially coronary heart disease on HRQoL while weaker predictors were MicroCx, older age, women, lower education, lower income, normotensive and high HbA1c [28].

This study reveals that being diagnosed with dyslipidaemia could be more deleterious to YQOL than being diagnosed with hypertension. Although the effects of hypertension were expected to be more detrimental to the patients' health than the effects of dyslipidaemia [32], it is intriguing to learn that T2D patients in this study perceived dyslipidaemia as worse than hypertension. It was possible to learn that dietary changes needed to control high cholesterol can be burdensome to some people and might be related to lower YQOL. Further study is needed to ascertain the possible reason adult patients with T2D chose dyslipidaemia and not hypertension on the YQOL. The negative effects of having dyslipidaemia and higher Total-C on EQOL might arise when patients' desire for more physical activities were impeded. This might happen when patients were faced with non-conducive environment such as unhealthy weather, lack of sport facilities, and feeling unsafe when going out of the house. Past studies had also reported the prevalent association of high level clinical parameters such as HbA1c, blood pressure and lipid with lower HRQoL [28, 32, 33]. However, it was 
not uncommon to find the non-association between these biomarkers and HRQoL, or psychosocial variables such as social support, self-esteem and psychological well-being [78-80] in past literature. This discrepancy might be due to the different HRQoL measures used in the different studies [22].

It is difficult to hypothesise the negative effects of lower SBP, HDL-C, and OHA use on WHOQOL-BREF. For the BP target and HDL-C, it was possible to learn that that patients who had healthy diets and exercised regularly faced the physical and external barriers represented in the PQOL and EQOL domains. In the case of OHA use and better PQOL, it might be that the use of OHA had ameliorated the physical symptoms of uncontrolled diabetes mellitus leading to improved physical functioning of daily activities, sleep and concentration. Similar observations were reported in newly diagnosed Dutch patients in general practices who showed improved vitality scores and HRQoL following the first year treatment for their diabetes [81]. A recent systematic review suggested that a greater HRQoL could be gained if insulin or newer injectable agents (glucagon-like peptide agonists and analogues, amylin analogues) were added to the patient's medication regimen rather than another OHA (e.g., adding sitagliptin or pioglitazone to metformin only) [82]. Unobserved determinants could probably explain the associations between treatment targets and HRQoL, particularly the SBP and its positive effects on the EQOL, such as the different geographic and social factors [83], dispositional optimistic personality and goal adjustment [84]. Also, being less critical to other people's comments and having a could-not-care-less attitude towards one's health $[85,86]$. Alternatively, it is also possible that the indicators of the disease control and health were relatively less important as compared to MacroCx and/or MicroCx. the outcomes of poor disease control, on HRQoL [87] and hence resulted in the diverged associations between them.

\section{Diabetes-related distress, depressive symptoms and quality of life}

Both DRD and DS were associated with lower HRQoL, especially on the YQOL. A Chinese study reported that emotional distress was the most important explanatory factor for quality of life which amounted to 28.7-53.8 \% of the total variance [88]. However, this study showed that DS instead of DRD had a more consistent, negative, and independent effect across all the domains of HRQoL. Similar patterns of association were also reported by Carper et al. who stated that DS severity (assessed with Montgomery Asberg Depression Rating Scale) was associated with poorer HRQoL (measured with Quality of Life Inventory), specifically on the achievement, psychosocial growth and environment domains while
DRD was associated with poorer HRQoL on the achievement domain [89]. The results of the present study suggested that DRD and DS were related, but were distinct constructs [47] associated with the various aspects of HRQoL that were beyond demographic and clinical factors.

Sundaram et al. reported the pervasive effects of DS among adult patients with T2D on a number of QoL measures that included the generic health status (12item Short-Form Health Survey [SF-12] and EQ-5D) and diabetes-specific QoL (Audit of Diabetes Dependent Quality of Life) [90]. The degree of diabetes-specific QoL perception was reported to be associated with the severity of DS $(r=0.503 ; p<0.001)$ among Brazilian patients with T2D [78]. Similarly, DS (measured with SF-12 Mental Component Score) among the elderly German patients with T2D was one of the independent predictors for HRQoL [91]. Besides DS, other mental disorders such as anxiety and schizophrenia had also been reported to be significant predictors for poorer diabetes-specific QoL [92].

These findings confirm the past reports on the prevalent and intimate relationships between the domains of HRQoL and emotions (DRD and DS), psychological well-being, and social functioning in adult patients with T2D [28]. By providing effective psychological support for DRD and DS, HRQoL may benefit to a large extent. Since evidence showed that DRD had preceded DS [93], and that DRD was both milder and more common in primary care as compared to DS [94], it would be a wise therapeutic and preventive opportunity to intervene for DRD in order to reduce the DS and its distal adverse effects and complications. An intervention for a relatively non-complicated psychological disorder such as DRD could probably be addressed with a less complex programme and competently delivered by the paramedics.

\section{Limitations and strengths}

Adopting a generic measure for HRQoL would cause some limitations and bring different interpretations. It was possible for T2D patients to report high levels of HRQoL in general, but poor HRQoL meant the patients were impaired by diabetes [95]. During clinical consultations, when opportunities arise to tailor the treatments of each T2D patient for the purpose of improving the patient's HRQoL, it is essential to differentiate between the general QoL and disease-specific HRQoL because patient's needs might go unnoticed if the general wellbeing or QoL are the only outcomes measured, and if it appeared to be good [96].

The strength of this study includes the relatively large sample size with high response rate, and it also represents the study population to the study domain from the aspects of socio-demographic characteristics 
[97]. Another important strength of the present study is the use of a validated and specific measure of DRD.

\section{Conclusions}

The majority of adult patients with T2D at a primary care setting had a good overall HRQoL. The independent determinants for HRQoL concurred with many of the past studies. In addition, DRD was reported to have negative effects on HRQoL. Meanwhile, religiosity had positive influences on HRQoL. Adult patients with T2D who were men, non-Malay, unmarried, unemployed, from older age group, had longer duration of diabetes, dyslipidaemia, MacroCx, did not engage in frequent exercises per week, had higher levels of Total-C, who experienced DRD and DS had lower HRQoL needed additional supports. Timely and less complex psychological intervention at primary care level could be prioritised for the right and most needy adult patients with T2D to improve their life experiences and HRQoL.

\begin{abstract}
Abbreviations
AHA: Anti-hypertensive agents; BMI: Body mass index; DDS-17: 17-items diabetes distress; DK: Dengkil health clinic; DRD: Diabetes-related distress; DS: Depressive symptoms; EQOL: Environment quality of life; HDL-C: High density lipoprotein-cholesterol; HRQoL: Health-related quality of life; LDL-C: Low density lipoprotein-cholesterol; LLA: Lipid-lowering agents; MicroCx: Microvascular complications; MacroCx: Macrovascular complications; MREC: Medical Research Ethics Committee, Ministry of Health, Malaysia; OHA: Oral hypoglycaemic agents; OQOL: Overall quality of life; PHQ-9: 9-items Patient Health Questionnaire; PQOL: Physical quality of life; QoL: Quality of life; SK: Seri Kembangan health clinic; SL: Salak health clinic; SRQOL: Social relationships quality of life; TG: Triglyceride; T2D: Type 2 diabetes; WHOQOL-BREF: World Health Organization Quality of Life- Brief; YQOL: Psychological quality of life.
\end{abstract}

\section{Competing interests}

The researchers had declared that they have no competing interests. The study sponsor had no role in the design or conduct of the study, writing of this report, or the decision to submit it for publication.

\section{Authors' contributions}

The subject matter of this study was conceived by BHC. However, SMS contributed to the conception and design of the study. While, $\mathrm{CBH}$ contributed to the data collection. $\mathrm{BHC}$ was also involved in the analysis and interpretation of the data. Furthermore, BHC, SMS and SSG contributed to the drafting of the manuscript. All researchers whose names appeared as 'authors' in this study contributed to the refinement of the manuscript, and approved the final manuscript for the purpose of publication.

\section{Acknowledgements}

This study received its funding from the Research University Grants Scheme 2 (RUGS/04-02-2105RU). The researchers would like to acknowledge WHO for the use of WHOQOL-BREF. They would also like to express their sincere appreciation to Polonsky et al. for giving their permission for the use of the English and Chinese versions of DDS-17, Sherina Mohd-Sidik and Xiaonan Yu for the use of the Malay and Chinese versions of PHQ-9 questionnaires, respectively, the Sepang and Petaling District Health Offices and officers for their support in this study and the Director of General Health for giving his permission to publish this report. Also, a heartfelt thanks to Dr. Firdaus Mukhtar, Dr. Nor-Kasmawati Jamaludin, Dr. Noor-Hasliza Hassan and Dr. Fuziah Paimin for their input during the planning of this study and their assistance in data collection. Lastly, the researchers are entirely grateful to all the staff in the three primary healthcare clinics for their efforts in facilitating the data collection.

\section{Author details}

${ }^{1}$ Department of Family Medicine, Faculty of Medicine and Health Sciences, Universiti Putra Malaysia, 43400 Serdang, Selangor, Malaysia. ${ }^{2}$ Department of Psychiatry, Faculty of Medicine and Health Sciences, Universiti Putra Malaysia, Serdang 43400Selangor, Malaysia.

Received: 17 February 2015 Accepted: 17 November 2015

Published online: 24 November 2015

\section{References}

1. Hinder S, Greenhalgh T. "This does my head in". Ethnographic study of self-management by people with diabetes. BMC Health Serv Res. 2012;12:83.

2. Weaver RR, Lemonde M, Payman N, Goodman WM. Health capabilities and diabetes self-management: the impact of economic, social, and cultural resources. Soc Sci Med. 2014;102:58-68.

3. Koch T, Kralik D, Sonnack D. Women living with type II diabetes: the intrusion of illness. J Clin Nurs. 1999;8:712-22.

4. Stuckey HL, Mullan-Jensen CB, Reach G, Kovacs-Burns K, Piana N, Vallis M, et al. Personal accounts of the negative and adaptive psychosocial experiences of people with diabetes in the Second Diabetes Attitudes, Wishes and Needs (DAWN2) Study. Diabetes Care. 2014;37(9):2466-74.

5. Nicolucci A, Kovacs Burns K, Holt Rl, Comaschi M, Hermanns N, Ishii H, et al. Diabetes Attitudes, Wishes and Needs second study (DAWN2): cross-national benchmarking of diabetes-related psychosocial outcomes for people with diabetes. Diabet Med. 2013;30:767-77.

6. Naranjo D, Hessler DM, Deol R, Chesla CA. Health and psychosocial outcomes in U.S. adult patients with diabetes from diverse ethnicities. Curr Diab Rep. 2012;12:729-38.

7. Schabert J, Browne JL, Mosely K, Speight J. Social stigma in diabetes: a framework to understand a growing problem for an increasing epidemic. Patient. 2013;6:1-10.

8. Low LL, Tong SF, Low WY. Mixed feelings about the diagnosis of type 2 diabetes mellitus: a consequence of adjusting to health related quality of life. Coll Antropol. 2014;38:11-20.

9. West C, McDowell J. The distress experienced by people with type 2 diabetes. Br J Community Nurs. 2002;7:606-13.

10. Rasmussen NH, Smith SA, Maxson JA, Bernard ME, Cha SS, Agerter DC, et al. Association of $\mathrm{HbA1c}$ with emotion regulation, intolerance of uncertainty, and purpose in life in type 2 diabetes mellitus. Prim Care Diabetes. 2013;7:213-21.

11. Kneckt MC, Keinanen-Kiukaanniemi SM, Knuuttila ML, Syrjala AM. Self-esteem as a characteristic of adherence to diabetes and dental self-care regimens. J Clin Periodontol. 2001;28:175-80.

12. Kovacs Burns K, Nicolucci A, Holt Rl, Willaing I, Hermanns N, Kalra S, et al Diabetes Attitudes, Wishes and Needs second study (DAWN2): cross-national benchmarking indicators for family members living with people with diabetes. Diabet Med. 2013;30:778-88.

13. Rosland AM, Heisler M, Piette JD. The impact of family behaviors and communication patterns on chronic illness outcomes: a systematic review. J Behav Med. 2012;35:221-39.

14. Browne JL, Ventura A, Mosely K, Speight J. 'I call it the blame and shame disease': a qualitative study about perceptions of social stigma surrounding type 2 diabetes. BMJ Open. 2013;3:e003384.

15. Harvey JN, Lawson VL. The importance of health belief models in determining self-care behaviour in diabetes. Diabet Med. 2009;26:5-13.

16. Das-Munshi J, Stewart R, Ismail K, Bebbington PE, Jenkins R, Prince MJ. Diabetes, common mental disorders, and disability: findings from the UK National Psychiatric Morbidity Survey. Psychosom Med. 2007;69:543-50.

17. Gentili P, Maldonato A, Grieco R, Santini A. Influence of patients' representations and beliefs about diabetes and its treatment on their adherence to therapy. Diabetes Nutr Metab. 2001;14:140-52.

18. Rose M, Fliege H, Hildebrandt M, Schirop T, Klapp BF. The network of psychological variables in patients with diabetes and their importance for quality of life and metabolic control. Diabetes Care. 2002;25:35-42.

19. Ismail K, Sloggett A, De Stavola B. Do common mental disorders increase cigarette smoking? Results from five waves of a population-based panel cohort study. Am J Epidemiol. 2000;152:651-7.

20. Mohebi S, Azadbakht L, Feizi A, Sharifirad G, Kargar M. Structural role of perceived benefits and barriers to self-care in patients with diabetes. J Educ Health Promot. 2013;2:37. 
21. Bradley C, Speight J. Patient perceptions of diabetes and diabetes therapy: assessing quality of life. Diabetes Metab Res Rev. 2002;18 Suppl 3:S64-9.

22. Speight J, Reaney MD, Barnard KD. Not all roads lead to Rome-a review of quality of life measurement in adults with diabetes. Diabet Med. 2009;26:315-27.

23. Rubin RR, Peyrot M. Quality of life and diabetes. Diabetes Metab Res Rev. 1999;15:205-18.

24. Imayama I, Plotnikoff RC, Courneya KS, Johnson JA. Determinants of quality of life in adults with type 1 and type 2 diabetes. Health Qual Life Outcomes. 2011;9:115.

25. Misra R, Lager J. Predictors of quality of life among adults with type 2 diabetes mellitus. J Diabetes Complications. 2008;22:217-23.

26. Glasgow RE, Ruggiero L, Eakin EG, Dryfoos J, Chobanian L. Quality of life and associated characteristics in a large national sample of adults with diabetes. Diabetes Care. 1997;20:562-7.

27. Wexler D, Grant R, Wittenberg E, Bosch J, Cagliero E, Delahanty L, et al. Correlates of health-related quality of life in type 2 diabetes. Diabetologia. 2006:49:1489-97

28. Wandell PE. Quality of life of patients with diabetes mellitus. An overview of research in primary health care in the Nordic countries. Scand J Prim Health Care. 2005;23:68-74.

29. Solli O, Stavem K, Kristiansen IS. Health-related quality of life in diabetes: the associations of complications with EQ-5D scores. Health Qual Life Outcomes. 2010:8:18.

30. Huang MC, Hung $\mathrm{CH}$. Quality of life and its predictors for middle-aged and elderly patients with type 2 diabetes mellitus. J Nurs Res. 2007;15:193-201.

31. Bourdel-Marchasson I, Druet C, Helmer C, Eschwege E, Lecomte P, Le-Goff $\mathrm{M}$, et al. Correlates of health-related quality of life in French people with type 2 diabetes. Diabetes Res Clin Pract. 2013;101:226-35.

32. Papadopoulos AA, Kontodimopoulos N, Frydas A, Ikonomakis E, Niakas D. Predictors of health-related quality of life in type II diabetic patients in Greece. BMC Public Health. 2007:7:186

33. Kiadaliri AA, Najafi B, Mirmalek-Sani M. Quality of life in people with diabetes: a systematic review of studies in Iran. J Diabetes Metab Disord. 2013;12:54.

34. Al Hayek AA, Robert AA, Al Saeed A, Alzaid AA, Al Sabaan FS. Factors associated with health-related quality of life among Saudi patients with type 2 diabetes mellitus: a cross-sectional survey. Diabetes Metab J. 2014:38:220-9.

35. WHO Consultation. Definition, diagnosis and classification of diabetes mellitus and its complications. In: Part 1: diagnosis and classification of diabetes mellitus. Geneva: World Health Organization; 1999. Report no. 99.2.

36. Ministry of Health Malaysia. Management of Type 2 Diabetes Mellitus. Putrajaya: Technology, Health Section, Assessment Division, Medical Development; 2009.

37. American Diabetes Association. Standards of medical care in diabetes-2015. Diabetes Care. 2015;38:S1-93.

38. The WHOQOL Group. Development of the World Health Organization WHOQOL-BREF quality of life assessment. Psychol Med. 1998;28:551-8.

39. Skevington SM, Lotfy M, O'Connell KA. The World Health Organization's WHOQOL-BREF quality of life assessment: psychometric properties and results of the international field trial. A report from the WHOQOL group. Qual Life Res. 2004;13:299-310.

40. Hasanah Cl, Naing L, Rahman AR. World Health Organization quality of life assessment: brief version in Bahasa Malaysia. Med J Malaysia. 2003:58:79-88.

41. Polonsky WH, Fisher L, Earles J, Dudl RJ, Lees J, Mullan J, et al. Assessing psychosocial distress in diabetes: development of the diabetes distress scale. Diabetes Care. 2005;28:626-31.

42. El Achhab Y, Nejjari C, Chikri M, Lyoussi B. Disease-specific health-related quality of life instruments among adults diabetic: a systematic review. Diabetes Res Clin Pract. 2008;80:171-84.

43. Chew BH, Mukhtar F, Sherina MS, Paimin F, Hassan NH, Jamaludin NK. The 17-items diabetes distress scale: translation and validation of the Malay version. Malays Fam Physician. 2015;10(2):22-34. [In Press]

44. Ting RZ, Nan H, Yu MW, Kong AP, Ma RC, Wong RY, et al. Diabetes-related distress and physical and psychological health in Chinese type 2 diabetic patients. Diabetes Care. 2011;34:1094-6.

45. Kroenke K, Spitzer RL, Williams JB. The PHQ-9: validity of a brief depression severity measure. J Gen Intern Med. 2001;16:606-13.
46. Sherina MS, Arroll B, Goodyear-Smith F. Criterion validity of the PHQ-9 (Malay version) in a primary care clinic in Malaysia. Med J Malaysia. 2012;67:309-15.

47. Fisher L, Mullan JT, Arean P, Glasgow RE, Hessler D, Masharani U. Diabetes distress but not clinical depression or depressive symptoms is associated with glycemic control in both cross-sectional and longitudinal analyses. Diabetes Care. 2010;33:23-8.

48. Yu X, Tam WW, Wong PT, Lam TH, Stewart SM. The Patient Health Questionnaire-9 for measuring depressive symptoms among the genera population in Hong Kong. Compr Psychiatry. 2012;53:95-102.

49. van Bastelaar KM, Pouwer F, Geelhoed-Duijvestijn PH, Tack CJ, Bazelmans E, Beekman AT, et al. Diabetes-specific emotional distress mediates the association between depressive symptoms and glycaemic control in Type 1 and Type 2 diabetes. Diabet Med. 2010;27:798-803.

50. Shim YT, Lee J, Toh MP, Tang WE, Ko Y. Health-related quality of life and glycaemic control in patients with Type 2 diabetes mellitus in Singapore. Diabet Med. 2012;29:e241-8.

51. Clifford EL, Collins MM, Buckley CM, Fitzgerald AP, Perry IJ. Unhealthy days and quality of life in Irish patients with diabetes. PLoS One. 2013;8:e81102.

52. Chew BH, Khoo EM, Chia YC. Social support and glycemic control in adult patients with type 2 diabetes mellitus. Asia Pac J Public Health. 2011. [Epub ahead of print].

53. Chew BH, Khoo EM, Chia YC. Does religious affiliation influence glycaemic control in primary care patients with type 2 diabetes mellitus? Ment Health Fam Med. 2011;8:21-8

54. Lee YK, Low WY, Ng CJ. Exploring patient values in medical decision making: a qualitative study. PLoS One. 2013;8:e80051.

55. Misra R, Lager J. Ethnic and gender differences in psychosocial factors, glycemic control, and quality of life among adult type 2 diabetic patients. J Diabetes Complications. 2009;23:54-64.

56. Simmons D, Weblemoe T, Voyle J, Prichard A, Leakehe L, Gatland B. Personal barriers to diabetes care: lessons from a multi-ethnic community in New Zealand. Diabet Med. 1998;15:958-64.

57. Koch T, Kralik D, Taylor J. Men living with diabetes: minimizing the intrusiveness of the disease. J Clin Nurs. 2000;9:247-54.

58. Kring AM, Gordon AH. Sex differences in emotion: expression, experience, and physiology. J Pers Soc Psychol. 1998;74(3):686-703.

59. Cheah WL, Lee PY, Lim PY, Fatin Nabila AA, Luk KJ, Nur Irwana AT. Predictors of health related quality of life in older people with non-communicable diseases attending three primary care clinics in Malaysia. Malays Fam Physician. 2012;7:21-30.

60. Sazlina SG, Zaiton A, Nor Afiah MZ, Hayati KS. Predictors of health related quality of life in older people with non-communicable diseases attending three primary care clinics in Malaysia. J Nutr Health Aging. 2012;16:498-502.

61. Tan MC, Ng OC, Wong TW, Hejar AR, Anthony J, Sintonen H. The association of cardiovascular disease with impaired health-related quality of life among patients with type 2 diabetes mellitus. Singapore Med J. 2014:55:209-16.

62. Akinci F, Yildirim A, Gozu H, Sargin H, Orbay E, Sargin M. Assessment of health-related quality of life (HRQoL) of patients with type 2 diabetes in Turkey. Diabetes Res Clin Pract. 2008;79:117-23.

63. Koopmanschap M. Coping with Type II diabetes: the patient's perspective. Diabetologia. 2002;45:S18-22.

64. Coruh B, Ayele H, Pugh M, Mulligan T. Does religious activity improve health outcomes? A critical review of the recent literature. Explore (NY). 2005;1:186-91.

65. Oleckno WA, Blacconiere MJ. Relationship of religiosity to wellness and other health-related behaviors and outcomes. Psychol Rep. 1991;68:819-26.

66. Ellison CG, George LK. Religious involvement, social ties, and social support in a Southeastern Community. J Sci Study Religion. 1994;33:46-61.

67. Landis BJ. Uncertainty, spiritual well-being, and psychosocial adjustment to chronic illness. Issues Ment Health Nurs. 1996;17:217-31.

68. Narayanasamy A. Spiritual coping mechanisms in chronically ill patients. Br J Nurs. 2002;11:1461-70.

69. Shaw JL, Brown J, Khan B, Mau MK, Dillard D. Resources, roadblocks and turning points: a qualitative study of American Indian/Alaska Native adults with type 2 diabetes. J Community Health. 2013;38:86-94.

70. Dye CJ, Haley-Zitlin V, Willoughby D. Insights from older adults with type 2 diabetes: making dietary and exercise changes. Diabetes Educ. 2003;29:116-27. 
71. Jafari N, Farajzadegan Z, Loghmani A, Majlesi M. Spiritual well-being and quality of life of Iranian adults with type 2 diabetes. Evid Based Complement Alternat Med. 2014;2014:619028.

72. Lynch CP, Hernandez-Tejada MA, Strom JL, Egede LE. Association between spirituality and depression in adults with type 2 diabetes. Diabetes Educ. 2012;38:427-35.

73. Vilhena E, Pais-Ribeiro J, Silva I, Pedro L, Meneses RF, Cardoso H, et al. Psychosocial factors as predictors of quality of life in chronic Portuguese patients. Health Qual Life Outcomes. 2014;12:3.

74. Hayes C, Kriska A. Role of physical activity in diabetes management and prevention. J Am Diet Assoc. 2008;108:S19-23.

75. van der Heijden MM, van Dooren FE, Pop VJ, Pouwer F. Effects of exercise training on quality of life, symptoms of depression, symptoms of anxiety and emotional well-being in type 2 diabetes mellitus: a systematic review. Diabetologia. 2013;56:1210-25.

76. Zanuso S, Balducci S, Jimenez A. Physical activity, a key factor to quality of life in type 2 diabetic patients. Diabetes Metab Res Rev. 2009;25 Suppl 1:S24-8.

77. Raisch DW, Feeney P, Goff Jr DC, Narayan KM, O'Connor PJ, Zhang P, et al. Baseline comparison of three health utility measures and the feeling thermometer among participants in the Action to Control Cardiovascular Risk in Diabetes trial. Cardiovasc Diabetol. 2012;11:35.

78. Horvath Z, de Lijster J, Kupper N. The association between quality of life, depressive symptoms and glycemic control in a group of type 2 diabetes patients: comment on Papelbaum et al. Diabetes Res Clin Pract. 2011;93:e5-6.

79. Nozaki T, Morita C, Matsubayashi S, Ishido K, Yokoyama H, Kawai K, et al. Relation between psychosocial variables and the glycemic control of patients with type 2 diabetes: a cross-sectional and prospective study. Biopsychosoc Med. 2009;3:4.

80. Sundaram M, Kavookjian J, Patrick JH, Miller LA, Madhavan SS, Scott VG. Quality of life, health status and clinical outcomes in Type 2 diabetes patients. Qual Life Res. 2007;16:165-77.

81. Adriaanse MC, Dekker JM, Spijkerman AM, Twisk JW, Nijpels G, van der Ploeg HM, et al. Health-related quality of life in the first year following diagnosis of Type 2 diabetes: newly diagnosed patients in general practice compared with screening-detected patients. The Hoorn Screening Study. Diabet Med. 2004:21:1075-81.

82. Thorpe CT, Fahey LE, Johnson H, Deshpande M, Thorpe JM, Fisher EB. Facilitating healthy coping in patients with diabetes: a systematic review. Diabetes Educ. 2013;39:33-52.

83. Weng C, Coppini DV, Sonksen PH. Geographic and social factors are related to increased morbidity and mortality rates in diabetic patients. Diabet Med. 2000;17:612-7.

84. Wrosch C, Scheier MF. Personality and quality of life: the importance of optimism and goal adjustment. Qual Life Res. 2003;12 Suppl 1:59-72.

85. Naeem AG. The role of culture and religion in the management of diabetes: a study of Kashmiri men in Leeds. J R Soc Promot Health. 2003;123:110-6.

86. Leach MJ, Segal L. Patient attributes warranting consideration in clinical practice guidelines, health workforce planning and policy. BMC Health Serv Res. 2011;11:221.

87. Hickey A, Barker M, McGee H, O'Boyle C. Measuring health-related quality of life in older patient populations: a review of current approaches. Pharmacoeconomics. 2005;23:971-93.

88. Liu M, Tai Y, Hung W, Hsieh M, Wang R. Relationships between emotional distress, empowerment perception and self-care behavior and quality of life in patients with type 2 diabetes [Chinese]. J Nurs. 2010;57:49-60.

89. Carper MM, Traeger L, Gonzalez JS, Wexler DJ, Psaros C, Safren SA. The differential associations of depression and diabetes distress with quality of life domains in type 2 diabetes. J Behav Med. 2014;37:501-10.

90. Sundaram M, Kavookjian J, Patrick JH. Health-related quality of life and quality of life in type 2 diabetes: relationships in a cross-sectional study. Patient. 2009;2:121-33.

91. Maatouk I, Wild B, Wesche D, Herzog W, Raum E, Muller H, et al. Temporal predictors of health-related quality of life in elderly people with diabetes: results of a German cohort study. PLoS One. 2012;7:e31088.

92. Donald M, Dower J, Coll JR, Baker P, Mukandi B, Doi SA. Mental health issues decrease diabetes-specific quality of life independent of glycaemic control and complications: findings from Australia's living with diabetes cohort study. Health Qual Life Outcomes. 2013;11:170.
93. Skinner TC, Carey ME, Cradock S, Dallosso HM, Daly H, Davies MJ, et al. Depressive symptoms in the first year from diagnosis of Type 2 diabetes: results from the DESMOND trial. Diabet Med. 2010;27:965-7.

94. Fisher L, Gonzalez JS, Polonsky WH. The confusing tale of depression and distress in patients with diabetes: a call for greater clarity and precision. Diabet Med. 2014;31:764-72.

95. Kinmonth AL, Woodcock A, Griffin S, Spiegal N, Campbell MJ. Randomised controlled trial of patient centred care of diabetes in general practice: impact on current wellbeing and future disease risk. The Diabetes Care From Diagnosis Research Team. BMJ. 1998;317:1202-8.

96. Lindsay G, Inverarity K, McDowell JR. Quality of life in people with type 2 diabetes in relation to deprivation, gender, and age in a new community-based model of care. Nurs Res Pract. 2011;2011:613589.

97. National Diabetes Registry Report, 2009-2012. In: Feisul Ml, Azmi SE, editors. Volume 1. Kuala Lumpur: Non-Communicable Disease Section, Disease Control Division. Department of Public Health; Putrajaya 2013.

\section{Submit your next manuscript to BioMed Central and we will help you at every step:}

- We accept pre-submission inquiries

- Our selector tool helps you to find the most relevant journal

- We provide round the clock customer support

- Convenient online submission

- Thorough peer review

- Inclusion in PubMed and all major indexing services

- Maximum visibility for your research 